\title{
THE USE OF ONLINE FORUMS IN THE CLASS OF ENGLISH LITERATURE
}

\section{Adrian OȚOIU*}

\begin{abstract}
A hands-on approach to the use of the online forums, formerly known as bulletin boards, in the teaching of literature at both BA and MA levels. Advantages, pitfalls, caveats and a few tips and tricks inspired by the author's over ten years' experience managing three such forums.
\end{abstract}

Keywords: online forum, teaching literature, bulletin board

\section{The numbers}

Over the last fifteen years I have maintained three online forums related to the courses I have been teaching at the North University Centre in Baia Mare. Each was designed to meet the needs of a particular academic program.

The EngLit forum, accessible at http://englit.myforum.ro , is meant as a complement to the seminar work at BA level. It was created in May 2008 and has been active ever since. A similar forum had operated for about three or four years before this date, on a different platform (uv.ro), but it is no longer online, due to hacking.

The Canada-Film forum, accessible at http://canada-film.myforum.ro, was active for six years between 2009 and 2015 to the benefit of the MA students in Canadian Studies taking a course on Canadian Film.

The KidLitForum, accessible at http://kidforum.otoiu.com, active since July 2011, is meant for the MA students in the Children's and Young Adult Literature in English.

It hould be noted that all these forums are personal, rather than institutional instruments, which means that they only assist the courses taught by the present author, and thus they are not representative for all the subjects the students take in their respective specializations.

Technically these forums are all based on phpBB. The first two run on the somewhat obsolete version 2.0.21, on the servers of a free Romanianbased web hosting service at myforum.ro. The KidLitForum runs on the latest phpBB version 3.0.12 on a paid service offered by ipage.com, as a

\footnotetext{
* Assistant Professor PhD, Technical University of Cluj-Napoca, Faculty of Letters, 76 Victoriei Street, Baia Mare (adrian.otoiu@cunbm.utcluj.ro).
} 
subdomain to otoiu.com. From the administrator's point of view, there is a notable difference between running an obsolete version versus the latest one. While the former has a dated interface with missing facilities and zero tech support, the latter sports the newest interface, faster servers and a 24/7 tech support.

The three forums total over 6000 posts, written by over 660 students. Here is a breakdown of these numbers by forum, as of 8 December 2019:

\begin{tabular}{|l|c|c|c|c|}
\hline & Semesters & Users & Threads & Posts \\
\hline EngLit & 23 & 411 & 601 & 4316 \\
\hline KidForum & 17 & 178 & 357 & 1198 \\
\hline Canada-film & 12 & 77 & & 584 \\
\hline
\end{tabular}

The numbers above prove a fairly intense activity. The variations are commensurate with the number of years the forums have been in service. The average number of posts per semester ranges between 60 (Canada-Film) and 188 (EngLit). While the average posting rate is of about 9 posts per user, it should be noted that about $35 \%$ of the users are opportunistic users, that never wrote more than just a couple of posts.

\section{The Rationale}

The original idea behind these forums was that they should operate as an extension of our seminars in English Literature. There were three categories of students to benefit from this facility: (a) The shy students, who cannot summon the courage to speak up during a seminar; plus those that lack self-confidence in their abilities of public speakers. (b) The slow students, who may not have finished reading the bibliography for the current seminar, and who could benefit from an time extension. (c) Students that commute and those that have part-time jobs, who might not attend all of the seminars.

The forum was thus supposed to offer a safe haven, away from the commotion of the classroom, that might encourage the shy to respond to the seminar issues in writing rather in speech. It was also to offer a time reprieve to the procrastinators, and a space bridge to those living elsewhere.

Of these three categories, those that seem to have benefitted the most from the forum system where the reluctant speakers and, to some extent, the distant learners.

Statistically the ratio of MA students using the forum is larger than that of the BA students. This can be explained by some characteristics of our MA students, namely the fact that most of them have jobs that may not allow them to attend every seminar. Besides, a study made in May 2019 on the 42 MA students in Children's Literature indicates that only $34 \%$ of these 
live within $30 \mathrm{~km}$ from the university, whereas $40 \%$ live within 30 to 100 $\mathrm{km}$ from the campus, and $26 \%$ live in towns located more than $100 \mathrm{~km}$ away. Thus two thirds of our students face longer commutes and might have trouble attending the seminars.

\section{Implementation and rules}

The students' registration to these forums was initially entirely optional. However, the above-mentioned situation of the long-distance students in the MA programme has encouraged us to transfer more seminar activities to the online medium, Such is the case of mid-term papers being submitted entirely on the forum. This shift made necessary that all of MA students in these classes should register to the forum.

A few ground rules were formulated. Students' user names had to bear some resemblance to their actual names, so that the teacher might identify them. However, since the forum is a publicly visible medium, those students who wanted to protect their online identity were free to use just their first name, initials or allusive names. After 2018, these solutions appeared to be compliant to some extent with the new GDPR regulations.

Posting rules are plain: Each new seminar discussion should be opened as a new thread (or topic), under a clear self-explaining title. The replies of the subsequent responders should be posts within the same thread. Multiple questions should be numbered, so that the subsequent responders' replies migth be easily connected to the original questions. Corrections of the typos and factual errors can only be made within 24 hours since the posting (this is a default behaviour unde phpBB); errors discovered later could only be corrected in a new post.

Common sense rules about academic form also apply. Clear literary English should be used. Personal attacks and discriminatory remarks are inacceptable and may lead to a permanent ban from the forum. The source of quoted materials, as well that of images used, should be clearly indicated.

A common problem occurring every year is that of posts that have actually been copy-pasted - without the due bibliographical references from various online sources or from the so-called cheat mills. As the forums' official policy is one of zero tolerance towards plagiarism, such unfortunate attempts at academic fraud are duly exposed by the teacher in a subsequent post that explains the nature of the offense. At the same time, the offender is banned from the forum for one or two months; repeat offenders will earn a permanent ban. Those who object to this are explained that the forum is a further opportunity offered to them, and not a right, as the teacher's job description does not include the official duty to carry out supplemental work reading the forum, on top of his/her regular duties in class. 


\section{Appraisal}

Students are informed that the forum has never been meant to be a total substitute for class activity. It was meant to supplement seminar activity, and therefore it could never account for more than $25 \%$ to $35 \%$ of the seminar grade. Students who do not attend any of the seminars cannot expect to get a pass grade based on forum activity alone.

Conversely, the grading system for forum activity operates on a different scale. This means that the same answer given online will be given a lower number of points than the same answer given in class (thus the maximum of 8 points for an excellent seminar contribution is reduced to 4 or 5 when posted online). The explanation for this disparity is plain: according to our curricula our medium of instruction is the classroom, and not the cyberspace. Nor do we offer distance learning classes at this point. Besides, the online medium is open to abuse, as we may never be completely sure if the author of the post is not being advised by peers, or even worse, if they may not be substituted by some other colleague. Identity fraud might be the greatest vulnerability of this system. The potential for abuse is worrying, and yet it should not deter us from taking advantage of the possibilities of the online forum.

\section{Later developments}

The decade spent perusing hundreds of students' posts on the forum have revealed to me the great potential of this tool. Working together with the students, I have discovered and perfected several activities that boosted not only communication among students, but also, indirectly, student selfreliance and openness in the classroom. Here are a few examples:

Close reading. The teacher of literature knows how difficult it is to have meaningful discussions on an excerpt of literary text, especially within a seminar that is supposed to cover a whole novel or a long theatrical play. Some students might stumble at words or phrases they do not understand, others have trouble following the pace of the disussion. And usually the time constraints force us to either rush through the analysis or just dump it altogether.

When I first invited students to move the discussion online, their response was overwhelming. We started with simple questions: "give your favourite scene from Pride and Prejudice" or a revealing dialogue, or portrayal, and them moved to more sofisticated topics like "identify premonitory symbolism in Tess of the d'Urbervilles", or "monsters and free will in East of Eden." In class there was hardly any time to delve into the style, the tropes, or the granular level of these texts. However, when left alone to their devices - their books and computers -, students produced 
carefully chosen literary fragments, they used underlining and colours to mark relevant words in the text and they offered often profound and subtlyworded analyses. Once posted online, these became in turn the object of discussion, with some colleagues merely nodding, others adding more arguments, and others diverging.

The surprise was great especially when it occured in classes that used to be relatively inert and unresponsive in the classroom. Maybe the rhythm of the seminar had always been wrong, too fast-paced, involving always just the top performers and leaving the others on the sidelines. When online, however, the time factor was suspended, students could consult the text at whatever moment suited them and then created their own rhythm, that suited most. I should say that much of this evolution seems to be of a recent date, and I might speculate that the availabilty of larger screen smartphones and of cheap internet might be a factor in the students' returning to these texts more often, probably in the most diverse of circumstances.

Challenges against the clock. The seminar of Introduction to Narratology is entirely based on the close reading of texts. When carried out in the classroom, the 100 minutes of a regular seminar might allow the dissection of just three, maybe four, texts. The speed factor only benefitted the top $10 \%$ of students, while unfortunately leaving the others behind.

Moving most of this activity onto the forum proved to be extremely beneficial. The teacher posts a battery of texts ( 7 to 10 depending on the class size), each forming a distinct topic, each followed by a set of 5 to 8 questions about narrative voice, point of view, narrative reliability, time and reader response. The timing of the posting is generally announced in advance and so the students, from the comfort of their homes, rush to post the answers. The first posters will obtain a bonus, while those who provide valid textual clues to support their claims are also rewarded. Thus this competition is not just about speed, but also about accuracy, which means that the same text might be dissected by 6 or 8 or even 10 students until it is exhausted.

This type of activity requires a great deal of work from the teacher. At the same time it is also rewarding even for the less advanced students, who have to read the posts of their colleagues before merely agreeing (which would not bring them many points) or finding new arguments or nuances (which is duly rewarded).

Building a corpus of texts. Another application of the forum was that of the support for a mid-term exam for my course on the Metafictional Fairy Tale. After being given some landmarks of the folktale in Europe, after learning about Vladimir Propp or the ATU system for the classification of the fairy tales, the students were invited to sample the folk tradition of Europe and briefly analyse a tale of their choice. Instead of them just 
handing me papers that nobody else would ever read, I wanted their findings to be exposed for all to see.

To that effect, I posted online a long list of links to folk collections from all parts of Europe, ranging from Greece to Lapland and from Armenia to Scotland, most of them available on sites like archive.org. I also offered a template for their analyses. Then the tales and their discussion poured on the forum.

The next year, instead of placing the European Fairy Tales subforum into the archive (as I always do with last year's subjects), I left it open, allowing it to grow and be enriched with the current year's crop. A this point the three years' work makes up an impressive patchwork of ethnic tales.

Building up a gallery. In a much less analytical vein, students studying Animal Tales were invited to pool their resources to create a list of children's films about animals, from Tarzan to Mowgly, from Lion King to The Lorax. Pictures, posters and brief summaries have been piling up in a growing resource of kinematic bestiaries.

\section{Conclusions}

My decade-long experience with online forums enables me to maintain that there is a great potential in this online resource. Even if today, in the world of instant messaging, social networks, vlogging and online streaming, the online forum has lost a bit of its earlier appeal, it can offer an alternative space for discussion, far from the inhibitions of the traditional classroom. 